\title{
L'ethnologie, un métier du patrimoine ? Réflexions autour de la question du patrimoine culturel immatériel
}

Ethnology, a heritage profession? Some reflections around the notion of intangible cultural heritage

\section{Christian Hottin}

\section{OpenEdition}

Journals

Édition électronique

URL : http://journals.openedition.org/insitu/13633

DOI : 10.4000/insitu.13633

ISSN : 1630-7305

Éditeur

Ministère de la culture

Référence électronique

Christian Hottin, «L'ethnologie, un métier du patrimoine ? Réflexions autour de la question du

patrimoine culturel immatériel », In Situ [En ligne], 30 | 2016, mis en ligne le 03 août 2017, consulté le 21 avril 2019. URL : http://journals.openedition.org/insitu/13633 ; DOI : 10.4000/insitu.13633

\section{Ce document a été généré automatiquement le 21 avril 2019.}

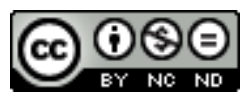

In Situ Revues des patrimoines est mis à disposition selon les termes de la licence Creative Commons Attribution - Pas d'Utilisation Commerciale - Pas de Modification 4.0 International. 


\title{
L'ethnologie, un métier du
} patrimoine? Réflexions autour de la question du patrimoine culturel immatériel

\author{
Ethnology, a heritage profession? Some reflections around the notion of \\ intangible cultural heritage
}

\section{Christian Hottin}

\section{Introduction}

\section{Les risques d'un titre}

1 Inclure dans un numéro d'In Situ consacré aux métiers du patrimoine un texte dédié à l'ethnologie et aux ethnologues peut avoir valeur de manifeste, à un moment ou cette discipline, après avoir été portée, dans les années 1980, par la création au sein du ministère de la Culture de la mission du Patrimoine ethnologique et par la constitution d'un maillage de conseillers en DRAC, est affectée par son invisibilité dans les organigrammes et par la lente décrue de ses effectifs dans les régions ${ }^{1}$.

2 Poser dans le titre un point d'interrogation quant à l'inclusion de l'ethnologie dans les métiers du patrimoine, c'est, déjà, apporter des éléments de réponse à ce reflux: la réponse ne va pas de soi, pas plus pour les ethnologues (ou devrait-on écrire «pour nombre d'entre eux » ?) que pour le reste de l'institution, tant l'ethnologie est absente des textes de loi, référentiels et normes professionnels, systèmes d'information qui sont non seulement les véhicules de la pensée institutionnelle, mais aussi la trame, la chair même de celle-ci. 
3 Associer dans un même titre "ethnologie» et "patrimoine immatériel ", c'est s'aventurer sur un terrain miné et s'exposer à de graves dommages, tant il est vrai que, depuis treize ans que cette convention existe, la notion de patrimoine culturel immatériel (PCI) a fait couler beaucoup d'encre (parfois sans grand discernement), a suscité maintes critiques (y compris, et des plus sévères, parmi les conservateurs du patrimoine) et causé maintes controverses dans le monde de l'ethnologie, dont la question, essentielle, de savoir si oui ou non les ethnologues sont plus que d'autres scientifiques concernés par cette question du patrimoine immatériel.

\section{Casus belli, rappel de quelques faits}

4 En 2003, l'Unesco adopte une convention dédiée à la sauvegarde des expressions culturelles traditionnelles. La France la ratifie en 2006 et s'engage dans sa mise en œuvre. Celle-ci est confiée par les responsables de la direction de l'Architecture et du Patrimoine à la mission du Patrimoine ethnologique (dite alors «mission Ethnologie ») établie en 1980 pour l'étude du patrimoine ethnologique via le développement de la recherche en ethnologie de la France et la professionnalisation des acteurs de ce secteur.

$5 \mathrm{Si}$, ainsi posée, la relation établie entre la mission et la convention peut paraître simple, elle l'est beaucoup moins dans son application. Non seulement parce que patrimoine « ethnologique » et patrimoine "immatériel», tout en se recoupant largement, ne se superposent pas ; Mais encore parce que la similitude des objets ne signifie pas l'identité des prises : le patrimoine ethnologique relève surtout du développement de la recherche, quand le PCI vise une sauvegarde globale qui fait appel à la participation. Et enfin parce que les ethnologues confrontés à cette convention ne vont absolument pas se comporter de la même manière que les autres professions liées au patrimoine face à un texte chargé d'une haute valeur juridique (traité multilatéral) et posant une injonction à la sauvegarde. En effet, leur rapport au patrimoine - le concept comme l'institution - est doublement distant: d'une part, dans leur grande majorité, les ethnologues en France ne sont pas issus de l'institution patrimoniale mais du monde de la recherche académique et sont accoutumés à travailler avec le ministère sans en dépendre, d'autre part, pour l'immense majorité d'entre eux, le patrimoine est un objet de recherche et non une cause à servir.

\section{Remonter le temps et disséquer les postures}

6 Tenter d'expliciter la complexité de cette relation entre une discipline (l'ethnologie), ses membres (les ethnologues) et un dispositif (la convention de l'Unesco pour la sauvegarde $\mathrm{du}$ PCI) sera notre propos. Il nous faudra pour cela remonter loin dans le temps, avant même la création de la mission du Patrimoine ethnologique, pour essayer de comprendre pour quelles raisons la relation nouée en France entre ethnologie et PCI ne peut se faire autour de la notion de « folklore » comme on l'a vu dans différents pays.

7 Il faudra ensuite revenir sur l'histoire de la mission du Patrimoine ethnologique, sur la professionnalisation des ethnologues à travers elle et sur quelques-uns des débats intellectuels qui ont parcouru cette " communauté de solitaires ", selon le mot de Gérard Lenclud, depuis cette fondation. On comprendra mieux alors dans quel contexte a été reçue, en 2006, la notion de patrimoine immatériel et pour quelle raison l'assimilation entre ethnologie et PCI, simple aux yeux de l'administration du patrimoine, a pu se 
révéler suffisamment problématique dans l'esprit de certains ethnologues pour susciter les critiques les plus acerbes.

On pourra alors détailler le panel des modes d'implication (ou de non-implication) des ethnologues dans le domaine du patrimoine, du rejet pur et simple à l'implication sans états d'âme au nom de la continuité des notions de "patrimoine ethnologique » et «patrimoine immatériel », avant de revenir plus longuement sur l'importance cruciale de la constitution du patrimoine immatériel comme objet d'étude. Aux côtés de cet apport particulièrement enrichissant, on offrira quelques vues sur les recherches développées par d'autres disciplines (droit, économie, sciences de l'information), qui toutes concourent à construire une approche française de la notion de patrimoine immatériel qui tente de croiser action et réflexion, centrée sur la référence à l'ethnologie, mais mobilisant cette discipline selon différentes approches.

\section{Scrupules méthodologiques}

9 Sans doute, ces interrogations restent-elles marquées par une vision du patrimoine en France qui a fait la part belle à l'État et à ces savants de formation attachés à son service que sont les conservateurs...Tout en manifestant une méfiance certaine envers les acteurs patrimoniaux étrangers à lui (ou qui, comme les chercheurs professionnels, entretiennent avec la puissance publique une relation ambiguë). Sans doute sont-elles aussi tributaires du point de vue de l'auteur : quelque distance critique qu'on revendique vis-à-vis de la politique conduite par son institution, surtout lorsque celle-ci manifeste, comme ce fut le cas initialement vis-à-vis du patrimoine culturel immatériel, des réticences culturelles, on demeure tributaire des conditions de son expérience. De même, ce texte ne saurait être un traité général établissant des faits définitifs sur l'appréhension par la France du patrimoine immatériel. Tout comme les précédents², il constitue une forme de portrait, réalisé à un moment donné. L'évolution de la situation décrite est nettement perceptible si on la compare aux textes plus anciens.

\section{Le folklore, impossible science du PCI}

En France, le folklore n'a reçu, en tant que discipline, qu'une tardive et très partielle reconnaissance institutionnelle, bien vite ruinée par les compromissions de l'occupation allemande et du régime de Vichy33. L'emploi du terme est régi, aujourd'hui encore, par certaines postures dont il vaut mieux ne pas s'écarter si l'on souhaite préserver sa crédibilité scientifique : le folklore et les folkloristes peuvent certes constituer un bon objet d'étude pour un ethnologue, mais il ne saurait être question de se revendiquer comme tel. Le terme en lui-même paraît recouvrir des sens et des usages divers, mais formant entre eux un système cohérent. Si on emploie le mot pour qualifier une discipline, il s'agira d'une forme d'ethnographie de médiocre valeur scientifique, pauvrement outillée sur le plan conceptuel, attachée à des objets supposés, pour ceux qui s'en réclament, relever de la " tradition " - sans que cette notion soit mise en question ${ }^{4}$ et plus attentive, en définitive, à l'accumulation de données brutes faiblement contextualisées qu'à leur mise en perspective en vue d'une interprétation globale. Si l'on fait référence au folklore pour qualifier des objets ou des pratiques, il sera alors principalement question d'activités qui se déroulent selon des codes et des règles figés, supposées pour leurs interprètes relever une fois de plus de la "tradition", sans que, là 
encore, ces acteurs eux-mêmes aient conscience du caractère construit de celle-ci. La manifestation la plus achevée du folklore serait alors l'exécution d'une danse qualifiée de régionale, au son d'une musique et dans des costumes tout aussi " régionaux ", l'ensemble de la performance étant présenté comme un spectacle se déroulant si possible dans un contexte marchand ${ }^{5}$ et donnant lieu, pour ses acteurs à une rémunération. Dès lors, les activités rangées sous ce terme sont fortement dévalorisées et l'emploi du mot, comme substantif ou comme adjectif, est stigmatisant, voire disqualifiant, aussi bien dans le champ des pratiques artistiques que dans celui des activités savantes. Un groupe de musique ou de danse folklorique ne pourra attendre de l'institution culturelle ni conseil ni subside, et c'est vers le ministère de la Jeunesse et des Sports que se tournent du reste ces formations. Au sein du monde académique, qualifier un travail "d'inspiration folklorique » ou un collègue de «folkloriste » vaut anathème et le rend politiquement suspect.

11 Cet interdit majeur étant posé, et si pertinent que soit, dans d'autres contextes culturels l'assimilation du patrimoine immatériel au folklore, il faudra, en vue d'adapter le concept de l'Unesco au cadre français, recourir à des équivalents tels que «traditions vivantes » ou « musiques et danses traditionnelles » pour en désigner les pratiques. L'adaptation se fait alors sans trop de peine... Il en va tout autrement de la référence à une discipline scientifique, et la substitution de l'ethnologie de la France au folklore s'avère des plus délicates.

\section{L'ethnologie de la France et sa place au sein des institutions patrimoniales}

\section{Les rencontres de l'ethnologie et du patrimoine : le « moment ATP » et le « moment MPE »}

Les activités de construction et de conservation du patrimoine reposent couramment sur un équilibre entre l'action publique, garantie par le droit, et l'expertise scientifique. Selon cette perspective, l'ethnologie de la France a connu, avant l'entrée en scène du patrimoine culturel immatériel, deux moments de rencontre avec les institutions culturelles.

Le premier, avec la création du musée des Arts et Traditions populaires (ATP) en 1937 et les développements ultérieurs de ce projet jusqu'à l'inauguration des bâtiments définitifs en 1972, s'est étendu une longue séquence temporelle ${ }^{6}$. Au cours de celle-ci, le développement de la recherche en ethnologie de la France et de la conservation du patrimoine ethnologique national (alors principalement fondé sur la collecte et l'archivage des objets constitutifs de celui-ci) se sont accomplis au sein d'un musée laboratoire, en fait constitué d'un musée (où œuvraient des conservateurs chargés de conserver) et d'un laboratoire, le centre d'Ethnologie française (dont les chercheurs menaient des activités de recherche sur l'ethnologie de la France). Le dispositif était complété par une association, la Société d'ethnologie française, intimement liée aux deux autres et chargée notamment de publier la Revue des arts et traditions populaires, par la suite rebaptisée Ethnologie française. Toutefois, dès la fin des années 1970, alors que les nouveaux bâtiments du musée des Arts et Traditions populaires étaient inaugurés depuis peu, ce dispositif centré sur la collecte des objets et sur l'existence d'un laboratoire 
unique tend à s'essouffler'. En 1980, grâce à l'initiative d'Isac Chiva, un nouvel outil est mis en place au service de l'ethnologie de la France ${ }^{8}$ : la mission du patrimoine ethnologique (MPE). Il s'appuie sur une autre institution, tout nouvellement créée, la direction du Patrimoine, au sein du ministère de la Culture. Unissant les activités en matière de conservation des monuments historiques et de recherche en archéologie ou dans le domaine de l'Inventaire général, cette nouvelle administration se voit en outre dotée d'une mission dédiée au "patrimoine ethnologique ${ }^{9}$ ». Pour la première fois, le patrimoine est alors explicitement défini non seulement en termes d'objets, mais aussi de pratiques et de représentations, ouvrant la voie à une appréhension de sa dimension proprement immatérielle ${ }^{10}$. Peu nombreuse en hommes mais dotée de moyens très importants, la mission porte un projet qui vise - ambiguïté qui s'avérera par la suite redoutable - la constitution du patrimoine ethnologique par le développement de la recherche en ethnologie de la France ${ }^{11}$. Ce projet se développe selon deux axes principaux : d'une part la formation (soit pour une bonne part la professionnalisation des acteurs bénévoles et associatifs) et d'autre part la recherche (par le lancement de programmes de recherche aux thématiques larges et ambitieuses).

\section{Vers la professionnalisation, non sans limites}

14 Le dispositif mis en place s'accompagne de la création de postes d'ethnologues, soit au sein de la mission proprement dite (on parle alors de " chargés de mission»), soit au sein des directions régionales du ministère, dont les organigrammes sont alors en plein développement (ici "conseillers pour l'ethnologie », ou "ethnologues régionaux", lorsque cette fonction est assurée par le directeur d'une association soutenue par la DRAC et la région, comme c'est le cas en Basse-Normandie). Les recrutements se font sur des postes d'ingénieurs d'étude, filière "recherche» qui est bien implantée dans les nouveaux secteurs du patrimoine que sont l'archéologie et l'Inventaire général, mais qui est en revanche moins présente dans le secteur des monuments historiques ou dans des domaines assimilables au patrimoine (on est avant la création d'une direction les intégrant tous), tels que les musées ou les archives. Les profils des ethnologues recrutés sont divers, tout autant que les missions accomplies (suivi de programmes de recherche en administration centrale vs ethnologie appliquée puis impliquée en région) et les manières de les interpréter.

Il faut surtout souligner que, si les effectifs croissent de manière relativement régulière jusqu'au milieu des années $1990^{12}$, le poste de chef de la mission du Patrimoine ethnologique échappe toujours aux ethnologues : après Elizabeth Fleury, il n'est occupé que par des archivistes paléographes, tous conservateurs d'archives ${ }^{13}$ à l'exception de Michel Melot (conservateur des bibliothèques). On a pu expliquer cet " évitement ", plus " qu'exclusion », de la fonction par le fait que la mission ayant à gérer d'important crédits liés aux différents laboratoires, un ethnologue, nécessairement issu de l'un d'eux, ne pouvait en tenir les rênes. La méconnaissance du domaine aurait été, en quelque sorte, une garantie d'équité de la part du chef de la mission. En outre, en 1990, est créé un corps commun de conservateurs du patrimoine, rassemblant les différents métiers de la filière, et cette mise en place se fait sans l'ethnologie, tout comme cette dernière est absente de la liste des spécialités de recrutement à l'École du patrimoine, école d'application fondée en même temps que le corps ${ }^{14}$. Pour finir, dans le courant des années 1990, les ethnologues du ministère refusent une évolution vers un statut de conservateur, tant 
parce qu'ils craignent d'être assimilés contre leur gré à l'Inventaire général qu'au nom de la défense de leur spécificité de personnel de recherche, distinct du personnel scientifique (conservateurs) ou de documentation (chargés d'études documentaires).

\section{Un développement entravé}

16 Si la professionnalisation au sein de l'institution patrimoniale est bien réelle, elle demeure néanmoins limitée ${ }^{15}$ tant pour ce qui concerne le nombre de personnes concernées que pour ce qui a trait aux postes occupés. Les effets de la professionnalisation dans les domaines de l'archéologie et de l'Inventaire général seront nettement plus probants, concernant dans le second cas plusieurs centaines de personnes et plusieurs milliers dans le premier (création de postes d'ingénieurs et de conservateurs, archéologues travaillant au sein des collectivités territoriales, salariés de l'INRAP, archéologues œuvrant dans les musées). De même, dans le courant des années 2000, des conservateurs issus de l'Inventaire ou de l'archéologie parviennent à des postes de sousdirecteur ou de directeur dans l'institution du patrimoine. Si la professionnalisation des ethnologues au sein de l'Université, du CNRS ou des musées de société a tiré profit de l'action conduite par la mission du Patrimoine ethnologique, en revanche, l'évolution professionnelle des ethnologues travaillant au sein de l'administration même du patrimoine a été entravée par les effets conjugués du blocage de l'accès à la fonction de chef de mission, d'une grille indiciaire peu valorisante et d'un maillage territorial des postes n'autorisant qu'une mobilité géographique sans progression en termes de responsabilité (la mutation se fait d'un poste de conseiller vers un poste de conseiller dans une autre DRAC). Enfin, le statut d'ingénieur, s'il offre au ministère une prise directe avec les activités de recherche (il n'y a au ministère ni chargés de recherche ni directeurs de recherche), combine nécessairement celles-ci avec des fonctions d'administration scientifique. De fait, au cours des années 2000, les transferts vers d'autres corps (maîtres de conférences, professeurs des universités, conservateurs du patrimoine) ou les mises à disposition au CNRS furent fréquentes, réduisant progressivement l'assise d'une communauté déjà peu nombreuse au départ.

\section{Le tournant réflexif}

17 Surtout, dès le milieu des années 1990, une bonne part des ethnologues du ministère, en administration centrale comme dans les DRAC, rompt en douceur avec le postulat initial de la constitution d'un patrimoine ethnologique national via le développement de la recherche en ethnologie de la France, pour se tourner, dans une perspective critique et réflexive, vers une "ethnologie du patrimoine" où l'institution est désormais saisie comme objet d'étude ${ }^{16}$. Ce mouvement, initié à partir de 1997 au sein d'une association, le GARAE, dont le président, Daniel Fabre, est par ailleurs directeur d'études à l'EHESS à Toulouse, trouve son institutionnalisation à partir de 2000-2001 avec la création d'un laboratoire dédié à l'anthropologie de la culture, le LAIC (devenu LAHIC), dont Daniel Fabre est le directeur et Odile Welfelé, alors chef de la mission du Patrimoine ethnologique, la directrice adjointe. Nombreux sont les ethnologues du ministère à rejoindre le laboratoire, soit par affiliation, soit par voie de mise à disposition. Si plusieurs conseillers pour l'ethnologie rejoignent le laboratoire, ils sont quelques-uns à déplorer que le travail en DRAC ne permette pas une institutionnalisation de l'approche réflexive comparable à celle offerte par le LAHIC pour l'administration centrale ; d'autres, 
enfin, se tiennent à l'écart du mouvement, souhaitant continuer à se réclamer du "patrimoine ethnologique».

\section{La rencontre de l'ethnologie et du patrimoine culturel immatériel}

\section{Retour sur un texte fort}

La prise de position, solidement argumentée, publiée dans L'Homme par Christian Bromberger à la suite du colloque tenu à Cerisy en $2012^{17}$, montre assez bien qu'un ethnologue ayant travaillé longtemps avec la mission du Patrimoine ethnologique, (au point d'être vice-président du conseil du Patrimoine ethnologique, l'instance chargée d'orienter le travail de la mission) pouvait ne se reconnaître aucune affinité avec le patrimoine culturel immatériel, et même critiquer fortement cette notion, tout en regrettant le temps de la mission du Patrimoine ethnologique! Si l'analyse de Christian Bromberger reprend des critiques courantes sur le patrimoine culturel immatériel (l'artificialité de la distinction entre matériel et immatériel d'une part, l'instrumentalisation politique constante de la notion d'une autre), sa nostalgie de la mission du Patrimoine ethnologique, qui, selon ses propres termes, ne s'occupait pas que de patrimoine mais soutenait des recherches dans des domaines très variés de la discipline ${ }^{18}$, mérite que l'on s'attarde un peu sur ce paradoxe, et que l'on tente d'expliquer à travers lui pour quelles raisons la relation entre "ethnologie» et "patrimoine immatériel » est loin d'aller de soi, ce que l'esquisse d'une évolution de la profession au ministère a déjà pu laisser entrevoir, mais qu'il faut compléter ici par un retour sur la discipline.

\section{La difficile articulation de l'ethnologie et du patrimoine}

En effet, en 1980, lors de la création de la mission du Patrimoine ethnologique, le fait que développer la recherche en ethnologie de la France et constituer son patrimoine ethnologique aient pu relever de deux projets finalement assez différents n'avait semblet-il pas été perçu par les différents acteurs du projet. Au sein de la nouvelle direction du Patrimoine, l'ethnologie voisinait par exemple avec l'archéologie, un service au sein duquel le dialogue entre recherche scientifique et conservation du patrimoine était envisagé sur le même mode : constituer le patrimoine archéologique et simultanément développer et structurer la recherche dans cette discipline. De la même manière, l'entreprise de grande envergure de l'Inventaire général du patrimoine culturel (matériel) passait par le renforcement de la recherche (en histoire de l'art, principalement, mais aussi en histoire des techniques) en vue de la création d'un patrimoine de la nation établi désormais sur des bases scientifiques solides ${ }^{19}$. Dans ce contexte, le projet de la mission du Patrimoine ethnologique, pourtant chargé d'ambiguïté, ne choquait pas et paraissait même s'articuler heureusement aux autres. Il a toutefois évolué d'une manière très différente de celui de l'Inventaire ou de celui de l'institution archéologique. Ainsi, alors que la loi de 2001 sur l'archéologie préventive $^{20}$ a consacré l'efficience de la relation entre conservation du patrimoine et recherche scientifique, faisant de ceux-ci deux objectifs essentiels de la loi, la relation entre patrimoine et ethnologie n'a cessé, tout au long des années, de se distendre. Les 
thèmes des recherches soutenues financièrement par la mission du Patrimoine ethnologique en attestent, qui sont passés, en vingt ans, d'une inscription franche dans le projet patrimonial (savoirs et savoir-faire naturalistes populaires) à une approche critique de la relation à l'esthétique (2001), programme au cours duquel fut notamment subventionnée une recherche portant sur la pratique du «topless » sur les plages qui constituait sans doute la limite de ce que l'administration du patrimoine était capable de supporter en ce domaine ${ }^{21}$. De même, la ligne éditoriale de la revue Terrain, principale publication liée à la mission du Patrimoine ethnologique et censée au départ exposer les résultats de travaux menés dans le cadre des programmes de recherche (son premier sous-titre était du reste « carnets du patrimoine ethnologique »), n'a cessé au fil des ans d'autonomiser toujours plus sa ligne éditoriale, abandonnant au passage toute référence au cadre national comme au projet patrimonial, en abordant de plus en plus fréquemment des thématiques liées à l'anthropologie cognitive ${ }^{22}$. Quant aux ethnologues eux-mêmes, en tout cas ceux liés au ministère de la Culture et à la mission du Patrimoine ethnologique, on a vu qu'ils ont tantôt maintenu ce lien entre recherche ethnologique et constitution d'un patrimoine ${ }^{23}$, tantôt fini par se résoudre à entrer à l'Université ou au CNRS, lieux plus propices, somme toute, au développement d'une activité de recherche libérée du cadre patrimonial. De ce fait, la relation entre ethnologie et patrimoine culturel immatériel ne peut être que complexe, et souvent conflictuelle.

\section{Les ethnologues et le patrimoine culturel immatériel : tout un panel d'attitudes}

La mise en place de la convention pour la sauvegarde du PCI et les politiques qui en découlent ont suscité des comportements très variables au sein de la communauté ethnologique nationale, allant du rejet le plus virulent à l'adhésion la plus enthousiaste, en passant par toute une gamme d'attitudes intermédiaires, plus ou moins critiques. Il est particulièrement notable que ces divisions n'aient pas opposé d'une part les ethnologues travaillant au sein du ministère de la Culture ou des établissements culturels (qu'on aurait pu supposer, a priori, plus favorables à cette politique, dans la mesure où ils sont assujettis à un statut administratif, moins tournés vers la recherche fondamentale et plus directement en prise directe avec la mise en œuvre des politiques culturelles) et d'autre part les chercheurs académiques de l'Université ou du CNRS. C'est au contraire chez les premiers, y compris au nom de la défense du "patrimoine ethnologique », que se sont parfois manifestées les réactions de rejet les plus violentes.

\section{Refus et résistances}

Parmi les membres de la communauté qui marquent le plus nettement leur opposition, rares sont toutefois ceux qui, à l'instar de Christian Bromberger, l'on fait par écrit dans une revue prestigieuse. Il suffit la plupart du temps de prendre position oralement, dans le cadre d'un colloque ou d'un séminaire, ou même plus simplement, dans des conversations privées. Les motifs le plus souvent invoqués pour fonder leur appréciation sur le PCI tiennent au caractère peu pertinent de la distinction entre matériel et immatériel, à l'omniprésence supposée des intérêts politiques dans la convention (et l'on peut alors imaginer que le chercheur perdrait sa morale et son éthique en s'y impliquant) et au risque de fossilisation des cultures qui découlerait de leur patrimonialisation par 
l'Unesco ${ }^{24}$. S'y ajoutent des jugements de valeur sur la piètre qualité intellectuelle des réflexions et travaux produits par l'Unesco (qu'il est alors possible de qualifier de «folklore » avec tout ce que ce terme peut avoir de péjoratif dans le contexte intellectuel français) ou le refus pur et simple de prendre en compte le point de vue d'une organisation internationale, notamment en raison de sa propre qualification académique (" on n'a pas à écouter les diktats de l'Unesco », « avec la formation que j'ai, je ne peux pas faire n'importe quoi »).

\section{La convention comme outil pour la démocratie culturelle}

Les positions défendues par les autres chercheurs ouvrent quant à elles la voie à des formes de mise en œuvre de la convention qui ne s'inscrivent pas toujours stricto sensu dans le cahier des charges prévu par l'Unesco - la pratique de l'Inventaire demeure à bien des égards un repoussoir intellectuel - mais revendiquent, chacune à sa façon, leur fidélité par rapport à l'esprit de celle-ci. Parmi les manifestations les plus originales conduites dans cet esprit se trouve sans doute le projet de démocratie participative centrée sur le PCI et mené à bien, entre 2008 et 2011, par Jean-Louis Tornatore, alors enseignant chercheur à l'université de Metz avec Marina Chauliac et Noël Barbe, tous deux conseillers pour l'ethnologie au ministère de la Culture ${ }^{25}$. Cette démarche conçue comme résolument compréhensive et non normative a conduit les ethnologues à accompagner la désignation par les habitants d'un parc naturel régional des éléments du patrimoine immatériel qui leur apparaissaient comme significatifs de leur territoire. Elle a été largement médiatisée lors de rencontres scientifiques et culturelles et reste l'une des formes de mise en œuvre de la convention les plus "puristes »: présentée comme fidèle à l'esprit de la convention (la participation des communautés est au cœur du dispositif) plus qu'à sa lettre (la démarche ne s'inscrit ni dans le cadre de l'Inventaire national du PCI, ni dans un projet de candidature à l'Unesco) ${ }^{26}$. La participation des communautés, idée centrale de la convention, constitue aussi le principe de l'exposition organisée par Noël Barbe sur le territoire du parc naturel régional des Vosges du Nord: dans cette enclave alsacienne en terre lorraine, un appel est lancé à la population afin qu'elle prête pour une exposition des objets que les habitants jugent représentatifs de leur patrimoine immatériel, tout en expliquant la relation qui unit ce patrimoine à cet objet. La collecte a rencontré un succès assez modeste mais a permis une intéressante problématisation de la notion de patrimoine, élaborée à partir du point de vue des habitants du territoire et non selon des catégories savantes ${ }^{27}$.

\section{S'inscrire plus étroitement dans les dispositifs}

23 On peut toutefois avoir une lecture beaucoup plus classique de la convention et considérer la participation moins comme une fin en soi que comme un moyen de construire la sauvegarde du patrimoine culturel immatériel. Les outils mis à disposition par le texte international sont alors largement sollicités. Ils sont du reste conçus dans un esprit qui ne refuse pas l'apport de l'expertise savante mais circonscrit étroitement celleci à des fins de sauvegarde : étude, documentation recherche figurent en effet dans le panel des actions concourant à la sauvegarde selon l'article 2 de la convention. De fait, nombreuses ont été les candidatures pour l'Unesco préparées avec l'appui scientifique d'ethnologues ou d'ethnomusicologues : le tracé de charpente (avec François Calame), les savoir-faire de la dentelle d'Alençon (avec Pierre Schmit), le compagnonnage (avec 
Nicolas Adell-Gombert), le fest noz, le gwoka de Guadeloupe (avec Dominique Cyrille), le Biou d'Arbois (avec Noël Barbe et Flavie Aihaud, déposé en 2015 en vue d'un examen ultérieur) ou le carnaval de Granville (avec Pierre Schmit, déposé en 2015 pour examen en 2016) ont bénéficié d'une telle expertise, fournie soit par les ethnologues du ministère de la Culture travaillant dans une région, soit par des enseignants chercheurs de l'Université.

C'est toutefois dans le travail d'inventaire du patrimoine culturel immatériel, coordonné par le ministère de la Culture et de la Communication ${ }^{28}$, que cette coopération entre ethnologues et détenteurs de patrimoine immatériel s'est avérée la plus fructueuse. Cet inventaire français du patrimoine immatériel a fait couler beaucoup d'encre : de fait, lors des premières enquêtes, le primat était clairement donné à l'expertise du chercheur (ministériel ou universitaire) ${ }^{29}$, tandis que le rôle des détenteurs de patrimoine culturel immatériel restait celui, classique en ethnologie, d'informateurs ${ }^{30}$. La pratique a sensiblement évolué au cours du temps, avec des projets d'inventaires de plus en plus souvent confiés à des associations ou centres culturels directement au contact des communautés (c'est par exemple le cas des différentes enquêtes conduites par l'Institut occitan de Pau sur le patrimoine culturel immatériel en Aquitaine ${ }^{31}$ ), ou encore menés par les associations de praticiens et détenteurs de PCI, l'appui fourni par les ethnologues se concentrant sur la méthodologie: particulièrement révélateur de ce nouveau mode opératoire est le travail sur les jeux et sports traditionnels en Bretagne, menés par la confédération FALSAB (jeux et sports traditionnels) et la fédération de Gouren (lutte bretonne), avec l'appui scientifique de l'université de Nantes et de Laurent Sébastien Fournier, spécialiste du sport et fin connaisseur de la convention de l'Unesco ${ }^{32}$.

\section{De l'ethnologie du patrimoine vers un projet d'étude interdisciplinaire}

\section{Le tournant réflexif appliqué à l'immatériel : ethnologie du PCI}

S'il est exact que la ratification de la convention par la France est intervenue à un moment où la notion de patrimoine ethnologique avait perdu pratiquement toute consistance intellectuelle et administrative ${ }^{33}$, elle s'est en revanche produite alors que l'ethnologie du patrimoine était en plein essor. En effet, à partir des premières années du $\mathrm{xxI}^{\mathrm{e}}$ siècle, le ministère de la Culture a fortement encouragé le développement de programmes de recherche invitant les ethnologues à se saisir du patrimoine comme d'un objet d'étude. En 2001, ces différentes initiatives ont été regroupées grâce à la mise en place d'un nouveau laboratoire, réunissant des professionnels de l'ethnologie du CNRS, de l'EHESS et du ministère de la Culture, le LAHIC. Les premières enquêtes de ce nouveau laboratoire, dont la chef de la mission Ethnologie au ministère occupait initialement le poste de directeur adjoint, ont porté sur des catégories de patrimoine bien établies et connues de tous: les monuments historiques, l'archéologie, les musées de société ou encore les archives ${ }^{34}$. En 2006, immédiatement après la ratification française, l'insertion du patrimoine immatériel dans le programme du laboratoire s'est concrétisée par l'instauration d'un séminaire, dont Chiara Bortolotto, chercheuse au LAHIC, et Sylvie Grenet, chargée de mission au ministère, ont assuré la coordination ${ }^{35}$. Ce séminaire a duré trois ans. Il a constitué une expérience particulièrement originale, pour l'administration du patrimoine, de mise en perspective critique de ses propres actions (on pense ici, par 
exemple à l'intervention de Jean-Louis Tornatore sur la " prise » française du patrimoine culturel immatériel), en même temps qu'une première ouverture internationale sur les recherches et actions conduites au titre du PCI. C'est également en son sein, dans un contexte neutre, moins passionnel que les arènes politiques ou administratives, qu'ont été présentés pour la première fois les arguments des promoteurs du très médiatisé « repas gastronomique des Français ». En 2011, les résultats ont été publiés en un ouvrage collectif de la collection " cahiers d'ethnologie de la France ", sous la direction de Chiara Bortolotto ${ }^{36}$. Ainsi, la première initiative soutenue et conduite par le ministère de la Culture ne fut ni un programme d'inventaires du PCI, ni une candidature à l'Unesco, mais bien un atelier de recherche collectif au sein duquel les politiques du patrimoine immatériel, y compris françaises, étaient traitées sans complaisance aucune. Ce projet, tout comme d'autres, a montré tout l'intérêt que pouvait présenter le patrimoine immatériel en tant qu'objet de recherche pour l'ethnologie. Il peut être mis en relation, par exemple, avec celui conduit par l'université de Neuchâtel et notamment Florence Graezer-Bideau au moment de la mise en place de l'Inventaire suisse des traditions vivantes ${ }^{37}$. Il est aussi un marqueur, selon nous, de la complexité et des ambiguïtés du traitement du patrimoine culturel immatériel par les ethnologues : pour les membres de cette profession, prendre position par rapport au patrimoine culturel immatériel engage de manière très profonde celui qui s'y risque sur la vision qu'il a de son métier et de l'histoire de celui-ci, tout en le mettant potentiellement en danger vis-à-vis de ses collègues. Objectiver le patrimoine immatériel, en faire son terrain, et donc, éventuellement, faire son terrain du travail de ses collègues (si ceux-ci participent à la mise en œuvre de la convention) permet au chercheur de regagner la distance critique nécessaire à son travail et lui évite d'être " pris » à son tour dans le patrimoine, et donc, potentiellement, d'être à son tour catégorisé comme folkloriste...

\section{Vers l'interdisciplinarité : approches juridiques et économiques, d'autres regards de chercheurs sur le PCI}

26 En septembre 2012, un important colloque a été organisé au centre culturel de Cerisy-laSalle sur le thème du patrimoine immatériel ${ }^{38}$. La vivacité des échanges ${ }^{39}$ et la diversité des points de vue exprimés ont montré que, si forte qu'elle soit, la relation entre le patrimoine immatériel et l'ethnologie, entre une catégorie de patrimoine et une discipline scientifique de référence, ne pouvait être exclusive, tant il est vrai que se rejouent sur la scène du PCI les luttes incessantes et les rivalités d'écoles qui ont contribué à la fragmentation toujours plus grande du paysage anthropologique français et à sa fragilisation institutionnelle. De fait, l'étude du patrimoine immatériel gagne à être conduite dans une perspective interdisciplinaire. Certaines des communications de Cerisy ont ouvert la voie à une approche renouvelée du $\mathrm{PCI}$, en rupture avec le débat récurrent sur la légitimité de sa place au sein de la discipline ethnologique. Le premier apport fondamental est venu des juristes, notamment ceux œuvrant au sein du CECOJI, laboratoire du CNRS aujourd'hui intégré à l'Institut des sciences sociales du politique (ISP) de l'École normale supérieure de Cachan. En effet, si la distinction entre patrimoine matériel et patrimoine immatériel est importante, c'est bien en regard du droit, et non de l'anthropologie. Les réflexions développées par Marie Cornu ${ }^{40}$, Jérôme Fromageau ${ }^{41}$ et, plus récemment, Noé Wagener, ont bien montré la spécificité de la notion de patrimoine culturel immatériel par rapport à l'état du droit du patrimoine en France : un droit qui 
concerne les personnes plus que les objets, centré sur le rôle des communautés (avec toute la difficulté qu'il y a à en définir les contours, tant dans l'espace social que dans la durée), et qui pose d'importants problèmes en matière de propriété intellectuelle ou industrielle. Ces analyses permettent notamment de mieux comprendre les difficultés culturelles qu'éprouve l'institution patrimoniale, et notamment des conservateurs, à intégrer le patrimoine culturel immatériel et ses éléments dans l'espace des patrimoines possibles: en effet, en 2015, le droit français du patrimoine ne reconnaît que le patrimoine matériel, composé de bien meubles ou immeubles ${ }^{42}$. Noé Wagener a en outre montré que le débat sur l'insertion de l'immatériel est loin d'être une nouveauté absolue dans le droit français, puisque, dès la fin des années 1930 par exemple, Georges Henri Rivière, fondateur du musée des Arts et Traditions populaires, avait plaidé - sans succès, il est vrai - pour la création d'une section des "monuments folkloriques» au sein de la commission des Monuments historiques. Il est tout aussi intéressant de souligner que la question de la participation des habitants à la désignation du patrimoine est une idée ancienne : dès la mise en place, en 1906, de la loi Beauquier ${ }^{43}$, consacrée à la protection des sites, avait été proposée la création de commissions composées de personnalités issues des territoires concernés par les projets de classements, dont les avis se seraient imposés aux préfets. Une fois de plus l'idée resta sans suite ${ }^{44}$. Les recherches en droit permettent en outre une approche comparée de la notion de PCI plus ample que celles précédemment menées et essentiellement centrées sur le caractère plus ou moins participatif des inventaires. À ce titre, dès 2013, le ministère de la Culture a conduit, avec le concours de Lily Martinet, avocate et doctorante en droit international, une étude comparée sur l'état du droit relatif au PCI dans soixante pays ayant ou non ratifié la convention ${ }^{45}$. Cette initiative avait un but essentiellement pratique, montrer le retard pris par la France en ce domaine face à des pays plus avancés, mais elle a trouvé depuis un prolongement théorique passionnant avec le programme d'échanges initié par l'Académie de la culture de Lettonie et l'ISP sous la direction d'Anita Vaïvade et Marie Cornu ${ }^{46}$.

Les sciences économiques semblent également très prometteuses pour la compréhension du patrimoine culturel immatériel et la mise en valeur des apports de la convention. Francesca Cominelli, maître de conférences à l'université Paris 1 , a consacré sa thèse à l'économie du patrimoine culturel immatériel en France, notamment à travers l'étude des savoir-faire traditionnels. Son travail a contribué à montrer là encore la spécificité du patrimoine immatériel par rapport au patrimoine matériel, et tout particulièrement en quoi le premier est une activité créatrice de richesses avant d'être un coût. Ces réflexions se sont prolongées dans un contexte plus international avec le troisième séminaire européen du Centre français du PCI, consacré en 2014 à l'économie du PCI. Il s'est agi de rompre avec des lieux communs bien enracinés: patrimoine immatériel et activité économique sont loin d'être incompatibles, du moment que celle-ci sert les communautés détentrices, mais le $\mathrm{PCI}$ ne saurait pour autant se réduire à un label, permettant de vendre un patrimoine comme une marque.

\section{Représenter les acteurs : de la liste à la carte, et la contribution des sciences de l'information}

L'originalité de ces approches pluridisciplinaires apparaîtra sans doute mieux encore, pour finir, à travers l'exemple d'un travail, celui de Marta Severo, qui croise recherches ethnologiques sur les pratiques du web et sciences de l'information. 
29 Selon le texte de la convention, les communautés jouent un rôle important dans la sauvegarde du patrimoine immatériel, à toutes les étapes de la chaîne patrimoniale, depuis l'identification jusqu'à la valorisation en passant bien entendu par la transmission. Pour autant, le terme de communauté n'est pas précisément défini par l'Unesco. On peut écrire, sans craindre de se tromper, qu'il y a en France, en 2015, soixante-cinq millions de détenteurs de patrimoine culturel, tout en gardant à l'esprit que chacune de ces personnes peut être détentrice d'un nombre quasiment infini de formes de patrimoine immatériel. Face à cette démesure s'est rapidement manifesté un besoin de mesure, ou, plus justement, de quantification des acteurs les plus pertinents de cette nouvelle politique, en prenant comme base les acteurs institutionnels concernés par la convention et les secteurs d'activité qui se rapportent aux cinq grands domaines de la convention visés à l'article 2 de celle-ci ${ }^{47}$. Cette recherche n'était pas une absolue nouveauté : dès 1983, le répertoire d'ethnologie de la France (ou repethno) avait été construit selon une logique similaire, face à la profusion des associations actives pour la sauvegarde de ce patrimoine ${ }^{48}$. En 2004, Portethno (portail d'ethnologie de la France) avait pris la suite de ce répertoire imprimét9. Toutefois, la cartographie des acteurs du patrimoine culturel immatériel impliquait d'autres choix et d'autres critères, en particulier la prise en compte d'organisations actives dans le champ des traditions vivantes, mais pas nécessairement dans l'étude et la valorisation de celles-ci (par exemple des associations sportives).C'est en 2010 seulement, soit quatre ans après la ratification, que le ministère de la Culture a mis en place un programme de recensement des acteurs du patrimoine culturel immatériel, confié à l'université de Bretagne occidentale (UBO) et plus particulièrement au Centre de recherche bretonne et celtique. Cette enquête a permis la constitution d'un annuaire d'environ 450 acteurs (institutions, sociétés, associations, fédérations, personnes répertoriées intuitu personœ). Il est apparu d'une réelle utilité pour diffuser des informations mais très insuffisant pour permettre une représentation des réseaux.

Aussi, à partir de 2010, la direction générale des Patrimoines a suivi avec un intérêt croissant les travaux menés par une jeune chercheuse en sciences de l'information de l'université de Lille 3, Marta Severo, pionnière en France dans la cartographie du web culturel ${ }^{50}$. Son projet, d'abord conduit de manière indépendante avec Chiara Bortolotto, a permis de cartographier les réseaux français du PCI sur Internet, faisant notamment ressortir la relative surreprésentation des acteurs institutionnels quand on compare le graphe français à celui des réseaux propres à d'autres pays (Italie et Suisse). À partir de 2014, le ministère de la Culture a engagé un partenariat avec le laboratoire Geriico de l'université Lille 3 : l'objectif de ce projet est de créer un observatoire des réseaux du PCI en France sur Internet, de rendre possible un suivi de l'évolution de ces réseaux et d'aider les différents partenaires du ministère à améliorer leur présence sur Internet. L'observatoire se met progressivement en place en $2015^{51}$.

\section{Conclusion}

31 L'ethnologie entretient, de fait, une relation complexe avec le patrimoine culturel immatériel. Elle peut fonctionner vis-à-vis de lui comme une discipline de référence, susceptible de décrire et d'expliquer les éléments relevant de cette catégorie de patrimoine mais elle peut aussi être mobilisée comme outil d'analyse du patrimoine culturel immatériel en tant que politique. Bien entendu, ce ne sont pas les mêmes ethnologues qui sont en mesure d'accomplir l'une et l'autre mission, tant elles relèvent de 
postures résolument différentes. Toutefois, c'est bien dans une approche clairement pluridisciplinaire des politiques du PCI, et non en faisant de ce dernier un simple objet d'étude supplémentaire pour l'ethnologie, que réside le positionnement le plus satisfaisant pour les services du ministère de la Culture en charge de la mise en œuvre de la convention : par les études juridiques, économiques et demain, sans doute, historiques, peut mieux être saisie la singularité du patrimoine immatériel par rapport au patrimoine matériel (et l'on voit alors que celle-ci ne réside pas uniquement dans la nature immatérielle des éléments considérés ou dans l'importance de la participation au cours des différentes étapes de la chaîne patrimoniale). Par une approche pluridisciplinaire et comparée, on peut en outre mieux percevoir la singularité française de l'appréhension du $\mathrm{PCI}$, et notamment les difficultés rencontrées par les administrations de l'État pour s'approprier ce traité entre États et les engagements qui en découlent.

\section{NOTES}

1. - Merci à Marie-Cécile Bouju ainsi qu'à Pascal Liévaux pour leur lecture attentive de ce texte. A l'occasion de mon départ de la direction générale des Patrimoines, je souhaite le dédier à tous les collègues ethnologues avec lesquels j'ai eu tant de plaisir à travailler dix années durant.

2. - Voir notamment, pour y repérer d'éventuelles contradictions avec ce qui sera exposé ici : HOTTIN, Christian. "Anti-monumental? Actualité du patrimoine culturel immatériel». Monumental, semestriel 1, 2008, p. 70-73, voir le site: http://www.iiac.cnrs.fr/IMG/pdf/ M08_IMMATERIEL_3.pdf [consulté le 22/08/2016] ; Id. « Entre ratification et inscriptions : la mise en œuvre d'une politique du patrimoine culturel immatériel en France (2005-2010) ». Terrain, dossier «Architecture et patrimoine ", $n^{\circ}$ 57, septembre 2011, p. 144-157. Disponible en ligne : http://terrain.revues.org/14390 [consulté le 22/08/2016]; Id. "À la recherche du patrimoine immatériel. Tâtonnements, tactiques et stratégies pour la mise en œuvre par la France de la Convention de 2003 ». Dans GAUTHIER, Antoine (dir.). Les mesures de soutien au patrimoine culturel immatériel : gouvernements, institutions, municipalités, Actes du colloque international, organisé par le Conseil québécois du patrimoine vivant, Québec, 14-18 avril 2011. Québec: CQPV, 2012, p. 87-107. Disponible en ligne: http://patrimonialisation/w-content/uploads/2009/10/Lesmesures-de-soutien-au-patrimoine-immateriel-actes-du-colloque-2011.pdf [consulté le 22/08/2016]; Id. "Le patrimoine culturel immatériel en France: des paradoxes évolutifs». L'Administration du patrimoine culturel immatériel en Europe : organisation, réglementation et procédures , actes du premier séminaire européen du patrimoine culturel immatériel organisé par la Maison des cultures du Monde (Centre français du patrimoine culturel immatériel) et la direction générale des Patrimoines (département du pilotage de la recherche et de la politique scientifique). Vitré : Centre français du patrimoine culturel immatériel, coll. «Cahiers du CFPCI, $1 », 2014$, p. 12-36, voir le site : http://www.cfpci.fr/medias/site/Cahier_CFPCI_n1.pdf [consulté le $22 / 08 / 2016]$.

3. - Voir CHRISTOPHE, Jacqueline, BOËLL, Denis-Michel et MEYRAN, Régis (dir.). Du folklore à l'ethnologie (avec une préface de Michel Colardelle et Isac Chiva). Paris : Éditions de la Maison des Sciences de l'Homme, 2009. 
4. - LENCLUD, Gérard. «La tradition n'est plus ce qu'elle était... ». Terrain [En ligne], 9 | octobre 1987, mis en ligne le 19 juillet 2007, consulté le 22 août 2016. URL : http://terrain.revues.org/3195 ; DOI : 10.4000/terrain.3195.

5. - «Cette manifestation typique du folklore breton fait désormais partie du patrimoine de l'Humanité ». Libération, 5 décembre 2012. Voir le site: http://next.liberation.fr/ culture/2012/12/05/le-fest-noz-breton-inscrit-au-patrimoine-de-l-unesco_865323 [consulté le 22/08/2016].

6. - Voir SEGALEN, Martine. Vie d'un musée. 1937-2005. Paris : Stock, 2005.

7. - Si les collections du musée des Arts et Traditions populaires forment aujourd'hui l'essentiel de celles du musée des Civilisations de l'Europe et de la Méditerranée à Marseille (MUCEM), le bâtiment parisien qui leur servait d'écrin demeure à l'abandon depuis leur déménagement, et son sort est des plus incertains. Triste fin pour une institution qui fut célébrée comme « Le Louvre du peuple».

8. - Voir : BENZAÏD, Redjem (dir.). Rapport sur l'ethnologie de la France. Besoins et projets. Paris : la Documentation française, 1979.

9. - BARBE, Noël. « Isac Chiva, ethnologie et politique patrimoniale ». Terrain [En ligne], 60 | mars 2013, mis en ligne le 19 août 2014, consulté le 18 décembre 2015. URL: http:// terrain.revues.org/15127; DOI : 10.4000/terrain.15127.

10. - La définition donnée dans le rapport mérite d'être citée : « Le patrimoine ethnologique d'un pays comprend les modes spécifiques d'existence matérielle et d'organisation sociale des groupes qui le composent, leurs savoirs, leur représentation du monde et, de façon générale, les éléments qui fondent l'identité de chaque groupe social et le différencient des autres. On y inclura donc: des agents : individus, groupes sociaux, institutions ; des biens matériels ou immatériels, œuvres virtuelles ou réalisées; des savoirs organisés; techniques, symboliques (magiques, religieux, ludiques) sociaux (étiquette, traditions de groupe), esthétiques; des moyens de communication : langues, parlers, systèmes de signes ». BENZAÏD, Redjem (dir.). Op. cit., p. 27.

11. - Voir le décret $n^{\circ} 80-277$ du 15 avril 1980 instituant un conseil du Patrimoine ethnologique : http://www.legifrance.gouv.fr/affichTexte.do?

cidTexte=LEGITEXT000006063221\&dateTexte=20090608 [consulté le 22/08/2016].

12. - En 1995 a lieu le dernier concours de recrutement d'ingénieurs d'étude, avec cinq postes. Les conseillers pour l'ethnologie sont alors présents dans une DRAC sur deux. À partir de 1997, à la suite de la fin de "l'exil de Babylone » de l'Architecture au ministère de l'Équipement, la nouvelle direction dite « de l'Architecture et du Patrimoine » (DAPA) privilégie le recrutement de conseillers pour l'architecture dans les directions régionales, au détriment de l'ethnologie.Un nouveau recrutement a toutefois eu lieu en 2006 (ingénieur de recherche) et un autre en 2014 (contractuel).

13. - Yves Renaudin, Gérard Ermisse, Jean-Marie Jenn, Odile Welfelé et Christian Hottin.

14. - Il est possible de passer le concours avec une option disciplinaire « ethnologie » mais cela ne débouche pas sur une filière professionnelle dans le domaine. Les ethnologues recrutés sont souvent dans les spécialités « musées » et " patrimoine scientifique technique et naturel ».

15. - La question de la professionnalisation des ethnologues a été étudiée de manière plus détaillée dans RAUTENBERG, Michel. « Mais pourquoi n'avance-t-on donc pas? Le surplace de la «professionnalisation » chez les ethnologues». Dans RAVIS-GIORDANI, Georges (dir.). Ethnologie (s). Nouveaux contextes, nouveaux objets, nouvelles approches. Paris : Éditions du CTHS, coll. «Le regard de l'ethnologue ", 2008, p. 83-99.

16. - Sur le tournant réflexif, voir TORNATORE, Jean-Louis. «La difficile politisation du patrimoine ethnologique ». Terrain [En ligne], $42 \mid$ mars 2004, mis en ligne le 9 septembre 2008, consulté le 19 janvier 2016. URL : http://terrain.revues.org/1791 ; DOI : 10.4000/terrain.1791. 
17. - BROMBERGER, Christian. " "Le patrimoine immatériel” entre ambiguïtés et overdose». L'Homme, 1/2014, $\mathrm{n}^{\circ} 209$, p.143-151. Voir le site: www.cairn.info/revue-l-homme-2014-1page-143.htm [consulté le 22/08/2016].

18. - «Paradoxe, cette «mission à l'Ethnologie» et la cellule qui l'a remplacée au sein du département du Pilotage de la recherche et de la politique scientifique (un pas de plus vers la marginalisation) se consacre au patrimoine et à l'histoire des institutions culturelles, alors que la «mission du Patrimoine ethnologique » impulsait des travaux d'ethnologie de la France centrés sur le présent (et son rôle pour le renouvellement de la discipline a été éminent)». Ibid., p. 143. Voir le site: http://www.cairn.info/revue-l-homme-2014-1-page-143.htm [consulté le 22/08/2016].

19. - On peut rappeler à ce propos que, en 1980, André Chastel, initiateur de l'aventure de l'Inventaire général, voyait en celui-ci l'avènement de l'âge «scientifique » du patrimoine. Selon André Chastel et Jean-Pierre Babelon, les monuments historiques, au XIX ${ }^{\mathrm{e}}$ siècle, n'en avaient été que l'âge «administratif ». BABELON, Jean-Pierre et CHASTEL, André. La notion de patrimoine [1981]. Paris : Liana Levi, 2000.

20. - Loi $\mathrm{n}^{\circ}$ 2001-44 du 17 janvier 2001 relative à l'archéologie préventive. Voir le site : http:// legifrance.gouv.fr/affichTexte.do?

cidTexte=JORFTEXT000000221337\&dateTexte=\&categorieLien=id [consulté le 22/08/2016].

21. - Voir KAUFMANN, Jean-Claude. Corps de femmes, regards d'hommes: sociologie des seins nus. Paris : Nathan, 1995.

22. - On comparera, par exemple : «Famille et parenté » ( $n^{\circ} 4$, mars 1985) et « Toucher » $\left(n^{\circ} 49\right.$, septembre 2007).

23. - C'est notamment le cas pour certains de ceux qui travaillent hors de l'administration centrale.

24. - Position qui rejoint de nombreuses critiques formulées dans la phase initiale de la convention. Voir BORTOLOTTO, Chiara. «Le trouble du patrimoine culturel immatériel ». Dans BORTOLOTTO, Chiara (dir.) avec la collab. de ARNAUD, Annick et GRENET, Sylvie. Le patrimoine culturel immatériel. Enjeux d'une nouvelle catégorie. Paris : Éditions de la Maison des sciences de l'Homme, 2011, p. 21-42.

25. - Il est intéressant de noter que cette démarche a été initialement présentée comme en rupture avec le «choix» de la mission Ethnologie de se lancer dans l'inventaire du PCI, l'inventaire étant présenté par Jean-Louis Tornatore comme un "déni de reconnaissance », à l'opposé de la démarche qu'il initie dans le PNR..

26. - Voir : BARBE, Noël, CHAULIAC, Marina et TORNATORE, Jean-Louis. «Intangible cultural heritage exposed to public deliberation: a participatory experience in a regional park ». Dans ADELL-GOMBERT, Nicolas, BENDIX, Regina F., BORTOLOTTO, Chiara, TAUSCHEK, Markus (éd.). Between imagined communities and communities of practice. Participation, Territory and the making of heritage. Göttingen Studies in Cultural Property, vol.8, 2015, p. 201-218, voir le site: http:// www.univerlag.uni-goettingen.de/bitstream/handle/3/isbn-978-3-86395-205-1/GSCP8_adell.pdf [consulté le 22/08/2016].

27. - Voir le site: http://www.alsace-bossue.net/upload/images/photos/pdf/ WEB_FLYER_PCI_ALSACE_BOSSUE.PDF [consulté le 22/08/2016].

28. - Voir les résultats de ce travail sur le site du ministère de la culture: http:// www.culturecommunication.gouv.fr/Politiques-ministerielles/Patrimoine-culturel-immateriel/ Inventaire-en-France [consulté le 22/08/2016].

29. - Ce fut le cas par exemple lors de l'enquête consacrée aux représentations et rituels liés aux arbres remarquables en Normandie. Voir : LEBORGNE, Yann. Des arbres, des rites et des croyances: un patrimoine culturel immatériel en Normandie. Caen/Bayeux: Centre régional de culture ethnologique et technique/Orep éd., 2012. 
30. - Chiara Bortolotto a pris elle-même part à ces enquêtes initiales et a pu à bon droit qualifier cette démarche « d'échec ». BORTOLOTTO, Chiara. «Identifier le patrimoine culturel immatériel en France. Réflexions d'un observateur participant ». Communication au colloque international «Définition, gestion et pratique du patrimoine culturel immatériel» organisé par l'Institut français de Hongrie, l'Office national hongrois du patrimoine culturel, l'université ELTE et le musée d'Ethnographie en plein air de Szentendre, Budapest et Szentendre, 23-25 septembre 2010 (actes non publiés).

31. - Voir à ce sujet le site de l'Institut occitan : http://www.in-oc.org/fr/services\#pci.

32. - Voir sur le site du MCC: http://www.culturecommunication.gouv.fr/Politiquesministerielles/Patrimoine-culturel-immateriel/Inventaire-en-France/Inventaire/Fiches-de-1inventaire-du-patrimoine-culturel-immateriel/Pratiques-sportives [consulté le 23/08/2016].

33. - On peut citer à l'appui de cette appréciation le jugement quelque peu sarcastique porté sur le patrimoine ethnologique et la mission par Noël Barbe et Jean-Louis Tornatore : «Cependant, là où il $\mathrm{y}$ avait un dispositif bourdonnant d'activité, ne subsistait qu'un dispositif très diminué, perdu dans les longs couloirs feutrés des «Bons Enfants». Que restait-il de la mission du Patrimoine ethnologique? Une adresse Internet: http://www.culture.gouv.fr/mpe ; «m-p-e », trois lettres qui permettaient d'ouvrir sur le site du ministère de la Culture et de la Communication la page de la mission à l'Ethnologie ».Consultable en ligne: http:// www.iiac.cnrs.fr/article887.html [consulté le 23/08/2016].

34. - Voir par exemple le remarquable dossier de la revue Histoire et société coordonné par Philippe Artières et Annick Arnaud: Lieux d'archive. Une nouvelle cartographie: de la maison au musée. Sociétés et représentations, $\mathrm{n}^{\circ}$ 19, avril 2005.

35. - Le programme du séminaire demeure consultable en ligne: http://www.iiac.cnrs.fr/ rubrique102.html [consulté le 23/08/2016].

36. - Voir : BORTOLOTTO, Chiara (dir.). Le patrimoine culturel immatériel. Op. cit.

37. - Voir les conclusions de ce travail : GRAEZER BIDEAU, Florence. «Inventorier les « traditions vivantes». Approches du patrimoine culturel immatériel dans le système fédéral suisse». ethnographiques.org, $\mathrm{n}^{\circ} 24$, juillet 2012. Ethnographies des pratiques patrimoniales: temporalités, territoires, communautés [en ligne]. (http://www.ethnographiques.org/2012/ Graezer-Bideau - consulté le 2.01.2016).

38. - Le programme est disponible sur le site du centre culturel de Cerisy. Les actes de ce colloque sont en cours de publication: http://www.ccic-cerisy.asso.fr/patrimoine12.html [consulté le 23/08/2016].

39. - Il faudrait envisager ici une histoire comparée de la mission du Patrimoine ethnologique, du musée des Arts et Traditions populaires (transformé en musée des Civilisations de l'Europe et de la Méditerranée, déménagé de Paris à Marseille et dont les collections historiques sont largement mises à distance, pour la plus grande partie d'entre elles, des espaces d'accueil du public) et la transmutation du musée de l'Homme en musée du Quai Branly. Sur ces deux histoires de musées, voir: MAZÉ, Camille, POULARD, Frédéric, et ROUSTAN, Mélanie (dir.). Les musées d'ethnologie. Culture, politique et changements institutionnels. Paris : éditions du CTHS, coll. «Orientations et méthodes, 24 ", 2013. Voir en outre L'ESTOILE, Benoît de. Le Goût des autres. De l'Exposition coloniale aux Arts premiers. Paris : Flammarion, 2007.

40. - Voir CORNU, Marie. «À qui appartient le patrimoine culturel immatériel ? ». Intervention au colloque «Le patrimoine culturel immatériel», centre culturel de Cerisy-la-Salle, 25 septembre 2012. Actes à paraître en 2016.

41. - Voir : CORNU, Marie, FROMAGEAU, Jérôme et HOTTIN, Christian (dir.). Droit et patrimoine culturel immatériel. [actes du colloque international organisé par le ministère de la Culture (DG Patrimoines), le LAHIC et le CECOJI (CNRS-université de Poitiers/UMR 6224, université Paris-Sud 11) au musée du Quai Branly, 13-14 janvier 2011]. Paris : L'Harmattan, coll. « Droit du patrimoine culturel et naturel», 2013. (Disponible en ligne sur le site des éditions L'Harmattan: http:// 
www.editions-harmattan.fr/index.asp?navig=catalogue\&obj=livre\&no=40821 [consulté le 23/08/2016].

42. - À ce titre, on peut avancer l'idée que vingt-cinq années d'École nationale du patrimoine puis d'Institut national du patrimoine, avec un enseignement exclusivement centré sur le traitement intellectuel ou matériel des biens meubles et immeubles ont sérieusement bridé chez les cadres supérieurs de l'institution la conception du patrimoine légitime - parce que légalement reconnu et consacré (le mot n'est pas trop fort) - au point que cette conception est moins large, moins ouverte à la nouveauté qu'elle ne l'était en 1980. « Naguère limitée aux biens culturels matériels et plus particulièrement, parmi ceux-ci, aux chefs-d'œuvre de l'architecture et de la peinture, la notion de patrimoine s'est élargie aux objets de la vie quotidienne, aux éléments plus humbles du cadre de vie et du travail, aux traditions orales et aux anciens modes de faire ». LECAT, JeanPhilippe. «L'année du patrimoine, communication faite à la séance du mercredi 29 octobre 1980 ». Paris : Institut de France/Typographie Firmin-Didot, 1980, p. 4.

43. - Voir : BARBE, Noël (dir.). Les mondes de Beauquier. Besançon : éditions du Sékoya, 2014.

44. - Voir: WAGENER, Noé. "La Convention de 2003 à l'aune du droit français: mise en perspective historique ». Communication à la journée d'études «Droit comparé du patrimoine culturel immatériel » organisée par l'Académie de la culture de Lettonie dans le cadre du projet Osmose (Académie de la culture de Lettonie - ISP), Riga, Académie de la culture, 29 juin 2015. Disponible en ligne : https://www.youtube.com/watch?v=CVyoKdfjbyk.

45. - Le résultat de cette enquête est disponible sur le site Internet du ministère de la Culture : http://www.culturecommunication.gouv.fr/Politiques-ministerielles/Patrimoine-culturelimmateriel/Vademecum/La-legislation-sur-le-patrimoine-culturel-immateriel-dans-le-monde [consulté le 23/08/2016].

46. - Pour une présentation du programme Osmose: https://dpc.hypotheses.org/category/ projets-collectifs/projet-osmose [consulté le 23/08/2016].

47. - Ce travail a été entrepris en 2011 dans le cadre d'un partenariat entre le ministère de la Culture et l'université de Bretagne occidentale (Centre de recherches bretonnes et celtiques) par Léna Le Roux et Marion Rochart. Il a été publié en ligne sur le site ministériel dédié au patrimoine culturel immatériel. Voir sur le site du ministère de la Culture: http:// www.culturecommunication.gouv.fr/Politiques-ministerielles/Patrimoine-culturel-immateriel/ Les-acteurs-du-PCI [consulté le 23/08/2016].

48. - Le repethno est ainsi défini : «Le Répertoire de l'ethnologie de la France est conçu pour offrir une vue géographique et synthétique de l'état des recherches en ce domaine. Il recense les chercheurs et les organismes dont les travaux se rattachent à la connaissance ethnologique de la France. Il expose leurs activités - qu'il s'agisse de recherche, de conservation, de documentation ou de diffusion -, constituant ainsi un instrument privilégié de liaison entre des personnes et des organismes aux objectifs diversifiés. ». Il peut être intéressant de se reporter à la première édition de ce répertoire, antérieure à la création de la mission du Patrimoine ethnologique : Répertoire de l'ethnologie de la France. Paris : 1979, ministère de la Culture et de la communication, Groupe de travail sur le patrimoine ethnologique et ministère des Universités, Laboratoire d'anthropologie sociale du Collège de France, de l'EHESS et du CNRS.

49. - Le projet Portethno a été conduit de 2004 à 2007, et l'initiative en revient à Odile Welfelé, alors chef de la mission du Patrimoine ethnologique. Les financements dévolus à ce projet ont été, à partir de 2008, réservés à la mise en place de l'Inventaire du patrimoine culturel immatériel en France. Portethno est toutefois toujours accessible en 2015. Après une révision globale en 2012, il s'est même enrichi d'une nouvelle rubrique, dédiée aux Archives de l'ethnologie de la France. Voir le site: http://www.culturecommunication.gouv.fr/Politiques-ministerielles/ Patrimoine-ethnologique/Travaux-de-recherche/Portethno [consulté le 23/08/2016].

50. - Une présentation de ses travaux est disponible sur son site personnel: http:// www.martasevero.com/ [consulté le 23/08/2016]. 
51. - Le projet est disponible en ligne: http://www.culturecommunication.gouv.fr/Politiquesministerielles/Patrimoine-ethnologique/Travaux-de-recherche/Etudes/Etude-d-observationdes-reseaux-du-patrimoine-culturel-immateriel-sur-internet [consulté le 23/08/2016].

\section{RÉSUMÉS}

Sans prétendre retracer ici la place de l'ethnologie et des ethnologues dans l'histoire récente de l'administration du patrimoine, on s'intéressera plus particulièrement à leurs réactions face à la mise en place, dans le milieu des années 2000, de la convention de l'UNESCO pour la sauvegarde du patrimoine culturel (PCI). Cette convention est ratifiée par la France en 2006 et conduit à une adaptation des habitudes de travail du service en charge de l'ethnologie au ministère de la Culture, lequel est appelé à travailler, traditionnellement, tant avec des ethnologues appartenant au monde de la culture qu'avec des chercheurs académiques. C'est tout un panel complexe d'attitudes qu'on tentera ici de présenter, depuis l'adhésion la plus franche aux principes et dispositifs de la convention jusqu'au rejet radical de celle-ci, en passant par différentes formes d'implications critiques. Sans prétendre encore une fois en rendre compte de manière exhaustive on cherchera à mettre ces comportements en perspective en revenant sur les relations entretenues par les ethnologues avec le patrimoine en 35 ans d'existence d'une politique de l'ethnologie au ministère de la Culture et de la Communication.

This article does not set out to retrace the place of ethnology and ethnologists in the recent history of France's heritage administration at the Ministry of Culture. Rather, it will take a closer look at how these ethnologists reacted to the drawing up, in the middle of the 2000s, of the UNESCO convention on intangible cultural heritage ('patrimoine culturel immatériel' in French, PCI). This convention was ratified by the French government in 2006 and led to a modification in the work habits of the service in charge of ethnology within the Ministry. Traditionally, this service was used to working with professional ethnologists in cultural spheres or in university contexts. The reactions to the UNESCO convention cover a complex range of attitudes, presented here, ranging from enthusiastic adhesion to the principles and operational features of the convention to their radical rejection and covering, in between, various forms of critical involvement. Without making any pretence at a complete survey, we will try to analyse these attitudes in the perspective of the relations between ethnologists and the heritage over the past 35 years during which the French ministry of Culture has had a policy for ethnology.

\section{INDEX}

Mots-clés : histoire culturelle, histoire des institutions, ethnologie, patrimoine, patrimoine culturel immatériel, histoire administrative, sciences sociales, mission du patrimoine ethnologique

Keywords : cultural history, history of institutions, ethnology, heritage, intangible cultural heritage, social sciences, 'mission du patrimoine ethnologique', ethnological heritage mission 


\section{AUTEUR}

\section{CHRISTIAN HOTTIN}

Conservateur en chef du patrimoine, Direction générale des Patrimoines, Ministère de la Culture et de la Communication christian.hottin@culture.gouv.fr 